\title{
Some possible factors affecting horse welfare assessment
}

\author{
Magdaléna Fejsáková1 ${ }^{1}$ Jana Kottferová1 ${ }^{1}$ Zuzana Dankulincová2, Eva Haladová1, \\ Raquel Matos ${ }^{1}$, Igor Miňo ${ }^{1}$ \\ ${ }^{1}$ University of Veterinary Medicine and Pharmacy in Košice, Department of Environment, Veterinary \\ Legislation and Economics, Košice, Slovakia \\ ${ }^{2}$ Pavol Jozef Šafárik University in Košice, Institute for Society and Health, Institute of Public Health, \\ Medical Faculty, Košice, Slovakia \\ Received January 17, 2013 \\ Accepted August 28, 2013
}

\begin{abstract}
The aim of this study was to assess the influence of various stimuli that confound interpretation of assessed indicators of horse welfare during rest and working period by the use of noninvasive methods of sampling. In total, 40 horses of different breeds and used for different purposes in Slovakia were used. The following indicators were tested: concentration of cortisol in saliva and 11,17-dioxoandrostanes in faeces measured by Elisa methods, heart rate and heart rate variability recording with the Polar Heart Rate Monitor and presence of stereotypical behaviour assessed with a horse questionnaire survey. The evaluated physiological responses were mostly affected by the type of work undertaken, especially horse movement intensity $(P<0.001)$ and horse equipment used during the working period $(P<0.01)$. Horses that showed stereotypical behaviour were less stressed during rest $(P<0.05)$ compared to horses without stereotypical behaviour. Horse breed, age, sex and stabling conditions affected only some of the heart rate indicators. The type of riding style had no fundamental influence on evaluated indicators. These observations highlight the difficulties in determining the welfare status in horses, since measurements can be affected by many factors that need to be investigated for achieving relevant outcomes. This is the first study in Slovakia focusing on the evaluation of horse welfare by non-invasive sampling.
\end{abstract}

Cortisol, 11,17-dioxoandrostanes, heart rate, stereotypes, non-invasive sampling

Horse welfare and stress-related disorders are becoming subjects of growing interest for increasing number of horse owners, breeders and riders. However, many problems are associated with the measurement of stress indicators, especially hormone concentrations in blood samples. Studies and manipulation with a live animal can affect the measured indicators and can thereby confound interpretation of the results. As there is a need to minimize study-induced disturbances, hands-off and non-invasive studies are often preferred (Moberg and Mench 2000). However, a number of biologically relevant issues (e.g. effect of sex, age, reproductive status, daily and seasonal patterns, effects of captivity, dietary effects, species-specific responses in stress responses) complicate the interpretation of animal welfare research results. These and many other confounding factors need to be investigated for the purpose of obtaining biologically relevant results. Factors affecting glucocorticoid concentrations in blood affect their concentration in saliva and faeces, too. These factors involve individual and interspecies differences, daily rhythms and seasonal patterns in secretion and the excretion of glucocorticoids, the effect of weather, sex, age and reproductive status, sensitization and habituation (Touma and Palme 2005).

The aim of this study was to determine the factors that can influence indicators of neuroendocrine and cardiovascular stress response and thus be potential sources of misleading results during the evaluation of horse welfare by non-invasive methods of sample collection.

Address for correspondence:

Magdaléna Fejsáková

University of Veterinary Medicine and Pharmacy in Košice,

Department of Environment, Veterinary Legislation and Economics,

Komenského 73,041 81 Košice, Slovakia
Phone: +421917171103

Fax: +42155671 1674

E-mail: fejsakova@gmail.com

http://actavet.vfu.cz/ 


\section{Materials and Methods}

Animals

Physically healthy horses ( $\mathrm{n}=40,25$ mares, 14 geldings and one stallion) of different breeds, mostly Slovak warm-blooded horses and their crossbreeds $(n=21)$ and crossbreeds of cold-blooded breeds $(n=6)$ were used in the study. From all tested horses, 13 were thoroughbred - American Quarter Horse $(n=4)$, Furioso $(n=2)$ and one horse from all next breeds: Shetland Pony, American Paint Horse, Arabian Horse, Holsteiner Horse, English Thoroughbred Horse, American Trotter and Czech Warmblood Horse. The age range of the tested horses was $1-25$ years $(10.25 \pm 5.50)$. The horses worked in hippotherapy or were used for recreational and low-grade competitive purposes. The horses belonged to different private owners who consented to all the procedures connected with experiment.

\section{Experimental design}

The effect of factors such as age, breed, physical activity, behaviour and type of horse equipment on the welfare indicators (salivary cortisol, 11,17-dioxoandrostanes - 11,17-DOA, heart rate - HR and heart rate variability - HRV) was evaluated in 40 horses during three testing days. Each horse was evaluated on a baseline day (Day 0 ) and the following day (Day 1). With regard to $24 \mathrm{~h}$ retarded occurrence of 11,17-DOA in horse faeces, this metabolite was evaluated on Day 1 as baseline day and also on the following day (Day 2). On Day 0, the horses were resting in their familiar home environment and were not used for any work. On Day 1, the horses were working in an area they were used to with their routine equipment and ordinary riding style. Testing periods lasted around $2 \mathrm{~h}$ on Day 0 and Day 1 and were performed during both testing days at the same times. On Day 2, the horses were reincorporated into their usual routine and had no further contact with researchers, only faeces samples were collected.

\section{Samples and analysis}

Saliva samples for the determination of basal cortisol concentration were taken at 30-min intervals during 2-h periods on Day 0. During Day 1, we collected a different number of samples depending on the number of riders, where saliva samples were taken before, during and after the riding period with one person. Saliva were sampled during the whole day (from 7:00 h to 21:00 h) as private horses had various training timetables. Saliva samples were collected using cotton rolls (Salivette Cortisol, Sarstedt, Germany). In the laboratory, the samples were stored at $-20^{\circ} \mathrm{C}$ until salivary cortisol determination by the competetive immunoenzymatic colorimetric method (CORTISOL SALIVA, DiaMetra, Italy). The average concentrations of salivary cortisol from each testing day were used for further analysis.

Faecal samples for analysis of cortisol metabolite 11,17-DOA were collected directly after defecation from the thoroughly mixed whole mass of the faeces at approximately the same time on Days 1 and 2. To prevent degradation, samples were transported in $2 \mathrm{ml}$ cryotubes in containers filled with liquid nitrogen (KB 7, Cryometal, Czech Republic). In the laboratory, samples were stored at $-80^{\circ} \mathrm{C}$ until analysis in the Institute of Biochemistry (Department of Natural Sciences) in Vienna using the method described by Möstl et al. (1999).

The heart rate was recorded on Days 0 and 1 with the Polar Heart Rate Monitor RS800 sd (Polar Electro Oy, Kempele, Finland). Data were downloaded from the receiver memory via Polar Irda USB Adapter to a PC and processed in Polar ProTrainer $5^{\mathrm{TM}}$ (Polar Electro Oy, Kempele, Finland). The following HR variables were quantified: minimum, maximum and mean HR (beat per minute - bpm), the root mean square of successive beatto-beat differences (RMSSD, ms) and mean beat-to-beat interval (R-R interval, $\mathrm{ms}$ ).

Two individuals (caretakers, riders or owners) most knowledgeable about the testing horse were independently asked to fill out the horse survey questionnaire. Questions were centred on basic information on the tested horse (age, sex, breed, temperament, incidence of stereotypic behaviour), history, care, and mostly used equipment.

\section{Statystical analysis}

Statistical analyses were carried out by the SPSS statistics package (SPSS, Version 16.0, Chicago, IL, USA) and Analysis of Variance (ANOVA). The significance of difference was checked by Mann-Whitney U test and Kruskal-Wallis $\mathrm{H}$ test. A $P$ value $<0.05$ was considered significant. Arithmetic means \pm standard deviations are presented.

\section{Results}

Horses younger than 6 years had a significantly higher mean heart rate on Day 1 (83.64 $\pm 19.95)$ and significantly lower R-R interval on both testing days (1190.38 \pm 219.83 and $875.31 \pm 219.82$ on Day 0 and Day 1, respectively) compared to horses older than 6 years (HR on Day $167.69 \pm 17.15$; R-R interval 1,352.81 \pm 211.70 and 1,060.78 \pm 241.94 on Day 0 and Day 1, respectively). Differences in other evaluated testing physiological welfare indicators were not significant (Table 1). 
Table 1. Statistical characteristic of indicators affecting horse welfare evaluation in the examined group of animals.

\begin{tabular}{lccccc}
\hline DAY 0 - resting period & Age & Sex $^{1}$ & Behaviour $^{1}$ & $\begin{array}{c}\text { Physical } \\
\text { activity }^{2}\end{array}$ & $\begin{array}{c}\text { Horse } \\
\text { equipment }^{2}\end{array}$ \\
\hline HR avg (bpm) Day 0 & $\mathrm{ns}$ & $\mathrm{ns}$ & $*$ & - & - \\
R-R interval avg (ms) Day 0 & $*$ & $* *$ & $*$ & - & - \\
RMSSD (ms) Day 0 & $\mathrm{ns}$ & $\mathrm{ns}$ & $\mathrm{ns}$ & - & - \\
Salivary cortisol (ng/ml) Day 0 & $\mathrm{ns}$ & $\mathrm{ns}$ & $*$ & - & - \\
11,17-DOA (ng/g) Day 1 & $\mathrm{ns}$ & $\mathrm{ns}$ & $\mathrm{ns}$ & - & - \\
\hline DAY 1 - working period & Age & Sex & Behaviour & Physical & Horse \\
& & & activity ${ }^{2}$ & equipment \\
\hline HR avg (bpm) Day 1 & $*$ & $\mathrm{~ns}$ & $* * *$ & $* *$ \\
R-R interval avg (ms) Day 1 & $*$ & $\mathrm{~ns}$ & $\mathrm{~ns}$ & $* * *$ & $* *$ \\
RMSSD (ms) Day 1 & $\mathrm{ns}$ & $\mathrm{ns}$ & $\mathrm{ns}$ & $\mathrm{ns}$ & $\mathrm{ns}$ \\
Salivary cortisol (ng/ml) Day 1 & $\mathrm{ns}$ & $\mathrm{ns}$ & $\mathrm{ns}$ & $* * *$ & $\mathrm{~ns}$ \\
11,17-DOA (ng/g) Day 2 & $\mathrm{ns}$ & $\mathrm{ns}$ & $\mathrm{ns}$ & $\mathrm{ns}$ & $\mathrm{ns}$ \\
\hline
\end{tabular}

HR - heart rate, R-R - beat to beat, RMSSD - root mean square of successive R-R differences, 11,17-DOA 11,17-dioxoandrostanes, bpm - beat per minute. ${ }^{1}$ Mann-Whitney U test, ${ }^{2}$ Kruskal-Wallis H test, ${ }^{*}$ significant at $P<0.05$, ** significant at $P<0.01$, *** significant at $P<0.001$, ns - nonsignificant

As horses were of different breeds, we compared evaluated indicators between warmblooded and cold-blooded breeds and between thoroughbred and crossbred horses with Mann-Whitney U test. Only one of the evaluated variables was affected by breed affiliation. RMSSD was significantly higher in cold-blooded horses compared to warm-blooded breeds on Day $1(P<0.05)$.

As only one of the tested horses was a stallion, this was removed from all analyses. Mares showed a higher mean heart rate and salivary cortisol and lower R-R interval and RMSSD during both testing days and higher 11,17-DOA on Day 2 compared to geldings. We validated signification only in R-R interval on Day $0(1,229.08 \pm 223.51$ and 1,425.64 \pm 182.01 for mares and geldings, respectively, Table 1).

Horses that showed stereotypical behaviour had a significantly lower mean heart rate $(46.33 \pm 11.34)$ and salivary cortisol concentration $(2.42 \pm 0.88)$ and significantly higher R-R interval $(1,393.53 \pm 261.13)$ compared to horses without stereotypical behaviour on Day 0 (HR 51.17 \pm 8.00 , salivary cortisol concentration $3.45 \pm 1.42$, R-R interval 1,243.92 \pm 183.95 , Table 1 ).

In relation to different kinds of work partaken in between the horses we tested, we compared evaluated physiological variables regarding the intensity of horse movement. Salivary cortisol concentration $(2.46 \pm 1.08,2.59 \pm 1.33$ and $3.82 \pm 1.25$ for walk, trot and gallop, respectively) and the mean heart rate $(60.95 \pm 16.07,75.25 \pm 10.31$ and 87.21 \pm 15.32 for walk, trot and gallop, respectively) were significantly ascended with more intensive movement, whereas R-R interval decreased significantly with more intensive movement $(1,140.25 \pm 233.76,987.00 \pm 155.68$ and $806.64 \pm 158.46$ for walk, trot and gallop, respectively; Table 1).

In respect of the different types of horse equipment we made a comparison among the evaluated physiological indicators in horses ridden with 3 different types of equipment. Horses that were ridden with the bosal, sidepull or hackamore had a significantly lower mean heart rate $(50.75 \pm 11.12)$ and a significantly higher $R-R$ interval $(1,253.80 \pm 185.43)$ compared to horses that were ridden with the halter $(78.43 \pm 18.21$ and $912.89 \pm 175.49$ 
for HR and R-R interval, respectively) and with the bridle or pelham (74.36 \pm 19.38 and $963.56 \pm 241.29$ for HR and R-R interval, respectively). Horses ridden with the bitless bridle had the lowest salivary cortisol concentration compared to horses ridden with other types of equipment; however, this difference was not significant (Table 1).

With ANOVA we found no differences between saliva specimens sampled at different time on Day $0(P=0.35)$ and Day $1(P=0.67)$. The obtained results do not confirm the existence of a daily rhythm of the cortisol concentration in horses.

\section{Discussion}

Young horses are more sensitive to novel situations than older. This may be the reason why in our research horses up to 6 years were more stressed according to their physiological reaction compared to older animals. On the other hand, physically and mentally mature animals can adapt to the environment and working conditions after certain period as shown by the physiological values in the evaluated indicators (Covalesky et al. 1992). More authors have described similar adaptations mostly in cortisol excretion associated with repeated stressor action in horses (Covalesky et al. 1992; Bachmann et al. 2003; Schmidt et al. 2010).

Although horse breeds are often described as having breed typical behaviours, temperament and personality (Lloyd et al. 2008), we found no significant validation in the physiological response for a fundamental effect of the horse breed on the evaluated indicators. Different values obtained in mares and geldings may be associated with more marked changes in the mares' hormonal status and behaviour.

Lower values of stress indicators in horses that showed stereotypical behaviour indicate that stereotypes can be beneficial to animals as a function of their adaptation to the living and working conditions (Mason 1991; Cooper and Albentosa 2005). Our results were validated significantly only on Day 0 (resting period), when the horses could express stereotypical behaviour more frequently than on Day 1 when the horses were tested during the working period and had less opportunity for free movement and unlimited behaviour. From our tested horses only $22.5 \%$ lived permanently in a group on pasture. Larger groups of horses lived in individual stalls with controlled and limited movement. This indicates that the social environmental conditions to which a horse is exposed may affect its ability to respond to challenging situations, altering its behaviour, physiology and compromising their welfare (Rivera et al. 2002).

Several authors have described increase in cortisol concentration and HR after physical activity in horses (Covalesky et al. 1992; Visser et al. 2002). This increase depends on the intensity and duration of the activity as well as on the condition and training of the horse (Collborn et al. 1991). Short exercise does not produce a real reaction to stress, which suggests that the saliva samples can be collected after a longer period following exercise (Strzelec et al. 2011). Individual horses can differ in their adaptiveness to environmental demands. Several studies on horses have showed that the mean HR was significantly higher in individuals characterized as more emotional (Visser et al. 2002).

The lowest salivary cortisol in horses ridden with the bitless bridle confirms the assumption that the bit can be a potential source of welfare problem in horses. Communication between the rider and the horse without the bit can be painless and stress-free (Cook 2007).

More authors have described cortisol natural circadian rhythm with the highest concentration in blood serum between 6:00 and 8:00 $\mathrm{h}$ and the lowest between 23:00 and 04:00 h (Hoffs is et al. 1970; Irvine a Alexander 1994). Circadian rhythm could be obliterated by the occurrence of short-term fluctuations, minor perturbation, effect of stress and how accustomed the horse is to its environment (Irvine and Alexander 1994). 
The results may help in selecting the appropriate experimental design and avoiding confounding factors in order to achieve meaningful and biologically relevant outcomes in the horse welfare assessment.

\section{Acknowledgements}

We would like to thank Prof. Dr. Erich Möstl for analysing cortisol metabolite concentrations in faeces. This work was supported by the Slovak Grant Agency KEGA - 012 - 003UVLF - 4/2010, VEGA 1/0052/10 and VEGA 1/0727/12 from the Ministry of Education, Science, Research and Sports of Slovakia.

\section{References}

Bachmann I, Bernasconi P, Hermann R 2003: Behavioural and physiological responses to an acute stressor in crib-biting and control horses. Appl Anim Behav Sci 82: 297-311

Collborn DR, Thompson DL, Roth TL, Capehart JS, White KL 1991: Responses of cortisol and prolactin to sexual excitement and stress in stallions and geldings. J Anim Sci 69: 2556-2562

Cook WR 2007: Bit - induced fear, Part 3: Use of the bit and bad behaviour.Vet Times 37: 36-38

Cooper JJ, Albentosa MJ 2005: Behavioural adaptation in the domestic horse: potential role of apparently abnormal responses including stereotypic behaviour. Livest Prod Sci 92: 177-182

Covalesky ME, Russoniello CR, Malinowski K 1992: Effect of show-jumping performance stress on plasma cortisol and lactate concentrations and heart rate and behavior in horses. J Equine Vet Sci 12: 244-251

Hoffsis GF, Murdick P, Tharp V, Ault K 1970: Plasma concentrations of cortisol and corticosterone in the normal horse. Am J Vet Res 31: 1379-1387

Irvine CHR, Alexander SL 1994: Factors affecting the circadian rhythm in plasma cortisol concentrations in the horse. Domest Anim Endocrin 11: 227-238

Lloyd AS, Martin JE, Bornett-Gauci HLI, Wilkinson RG 2008: Horse personality: Variation between breeds. Appl Anim Behav Sci 112: 369-383

Mason GJ 1991: Stereotypies: a critical review. Anim Behav 41: 1015-1037

Moberg GP, Mench JA 2000: The biology of animal stress. Basic principles and implications for animal welfare UK: CABI Publishing, 377 p.

Möstl E, Messmann S, Bagu E, Robia C, Palme R 1999: Measurement of glucocorticoid metabolite concentrations in faeces of domestic livestock. J Vet Med A 46: 621-632

Rivera E Benjamin S, Nielsen B, Shelle AJ, Zanella A 2002: Behavioral and physiological responses of horses to initial training: the comparison between pastured versus stalled horses. Appl Anim Behav Sci 78: 235-252

Schmidt A, Hödl S, Möstl E, Aurich J, Müller J, Aurich C 2010: Cortisol release, heart rate, and heart rate variability in transport-naive horse during repeated road transport. Domest Anim Endocrin 39: 205-213

Strzelec K, Kankofer M, Pietrzak S 2011: Cortisol concentration in the saliva of horses subjected to different kinds of exercise. Acta Vet Brno 80: 101-105

Touma C, Palme R 2005: Measuring fecal glucocorticoid metabolites in mammals and birds: the importance of a biological validation. Ann NY Acad Sci 1046: 54-74

Visser AK, Reenen CG, van der Werf JTN, Schilder MBH, Knaap JH, Barneveld A, Blokhuis BJ 2002: Heart rate and heart rate variability during a novel object test and a handling test in young horses. Phys Behav 76: 289-296 